\title{
Autoimmune encephalitis and epilepsy: evolving definition and clinical spectrum
}

\author{
Joo Hee Seo, MD¹, Yun-Jin Lee, MD, PhD ${ }^{1,2}$, Ki Hyeong Lee, MD¹, Elakkat Gireesh, MD¹, Holly Skinner, DO ${ }^{1}$, Michael Westerveld, \\ $\mathrm{PhD}^{1}$ \\ ${ }^{1}$ Comprehensive Epilepsy Center, AdventHealth for Children, Orlando, FL, USA; ${ }^{2}$ Department of Pediatrics, Pusan National University Children's Hospital, Pusan \\ National University College of Medicine, Yangsan, Korea
}

Advances in autoimmune encephalitis studies in the past 10 years have led to the identification of new syndromes and biomarkers that have transformed the diagnostic approach to the disorder. The disorder or syndrome has been linked to a wide variety of pathologic processes associated with the neuronspecific autoantibodies targeting intracellular and plasma membrane antigens. However, current criteria for autoimmune encephalitis are quite dependent on antibody testing and responses to immunotherapy, which might delay the diagnosis. This form of encephalitis can involve the multifaceted presentation of seizures and unexpected behavioral changes. The spectrum of neuropsychiatric symptoms in children is less definitive than that in adults, and the incorporation of clinical, immunological, electrophysiological, and neuroradiological results is critical to the diagnostic approach. In this review, we document the clinical and immunologic characteristics of autoimmune encephalitis known to date, with the goal of helping clinicians in differential diagnosis and to provide prompt and effective treatment.

Key words: Autoimmune encephalitis, Autoimmune epilepsy, Antineuronal antibodies, Immunosuppression therapy, Epilepsy in children

\section{Introduction}

Acute encephalitis is an inflammatory condition of the brain with multiple etiologies that has a crucial impact on relevant morbidity, occurring across all ages, although more common in children. ${ }^{1)}$ Encephalitis etiology remains unknown in $>50 \%$ cases. This explains why the criteria for encephalitis diagnosis are mainly based on infection characteristics. The Consensus Statement of the International Encephalitis Consortium reported that an encephalitis diagnosis required evidence of an altered mental status lasting $\geq 24$ hours with no alternative cause identified (major criterion) along with $\geq 2$ minor criteria, including fever $\geq 38^{\circ} \mathrm{C}$ ( 72 hours before or after presentation), generalized or partial seizures not fully attributable to a pre-existing seizure disorder, new-onset focal neurologic findings, cerebrospinal fluid (CSF) pleocytosis (white blood cell [WBC] count $\geq 5$ / $\mathrm{mm}^{3}$ ), abnormal brain parenchyma on neuroimaging, and abnormalities on electroencephalography (EEG) not ascribable to other causes. ${ }^{1}$ Two of these minor criteria were required for a possible diagnosis of encephalitis, while $\geq 3$ are needed for a probable or confirmed diagnosis.

However, the possible role of autoimmunity in encephalitis development has been reported in the last decade. ${ }^{2)}$ The most inspiring progress came with the discovery of multiple neuralspecific autoantibodies in patients with acute seizures and status epilepticus (SE) intractable to antiepileptic drug (AED) therapy. Increasing evidence supports an autoimmune etiology for seizures in the absence of these syndromic manifestations (e.g., limbic or extralimbic encephalitis) for a patient subgroup showing drug-resistant epilepsy. ${ }^{3,4)}$ Clinical hints include acute or subacute onset (over days to weeks), a remarkably high seizure frequency, seizure variability or multifocality, AED resistance, personal or family history of autoimmunity, and recent or past neoplasm history. ${ }^{3,4)}$ Rapidly evolving cognitive impairment, neuropsychiatric symptoms, evidence of multilevel central nervous system (CNS) involvement, or new-onset movement disorder propose but are not necessary for diagnosis.

Cases with an autoimmune pathogenesis have a wide spectrum of clinical manifestations; some features differ significantly from those that are usually found in infectious encephalitis. We aimed to report a case of confirmed anti- $\mathrm{N}$-methyl- $\mathrm{D}$-aspartate (NMDA) receptor encephalitis and to review the immunologic and clinical characteristics of autoimmune encephalitis to support clinicians with differential diagnosis and providing early effective treatment.

\section{Case}

A developmentally appropriate and previously healthy 15 -

Corresponding author: Joo Hee Seo, MD. Comprehensive Epilepsy Center, AdventHealth for Children, 615 E. Princeton Street, Orlando, FL 32803, USA 凶 E-mail: joohee.seo.md@adventhealth.com, https://orcid.org/0000-0002-6321-8766

Received: 30 May 2019, Revised: 23 July 2019, Accepted: 13 August 2019

This is an open-access article distributed under the terms of the Creative Commons Attribution Non-Commercial License (http://creativecommons.org/licenses/by$\mathrm{nc} / 4.0 /$ ) which permits unrestricted non-commercial use, distribution, and reproduction in any medium, provided the original work is properly cited.

Copyright (c) 2020 by The Korean Pediatric Society 
year-old girl presented to the Emergency Department with a 4-day history of severe and persistent headache associated with a 1-day history of photophobia, altered mentality, and gait disturbance. No recent contributing history was noticed. She was afebrile but had tachycardia (111 beats/min) and hypertension $(146 / 78 \mathrm{mmHg})$. She was $160 \mathrm{~cm}$ tall and weighed $51 \mathrm{~kg}$. The initial neurologic examination revealed lethargic mentality with emotional lability, dysarthria, and an ataxic gait without lowerextremity weakness; however, no focal neurologic deficits or meningismus-related signs were apparent. She had no palpable lymphadenopathy or rash.

The initial work-up showed normal complete blood count, electrolyte, and chemistry panel, and thyroid function test findings. Pregnancy testing was negative. Brain magnetic resonance imaging (MRI) showed unremarkable results. Lumbar puncture revealed significant lymphocytic pleocytosis (WBC, 114/ $\mathrm{mm}^{3}$; $86 \%$ lymphocytes); normal protein $(21 \mathrm{mg} / \mathrm{dL}$ ) and glucose (64 $\mathrm{mg} / \mathrm{dL}$ ) levels; and normal opening pressure. CSF meningitis and encephalitis panels were negative for multiple bacterial, viral, and fugal antigens, including herpes simplex virus (HSV)-1,

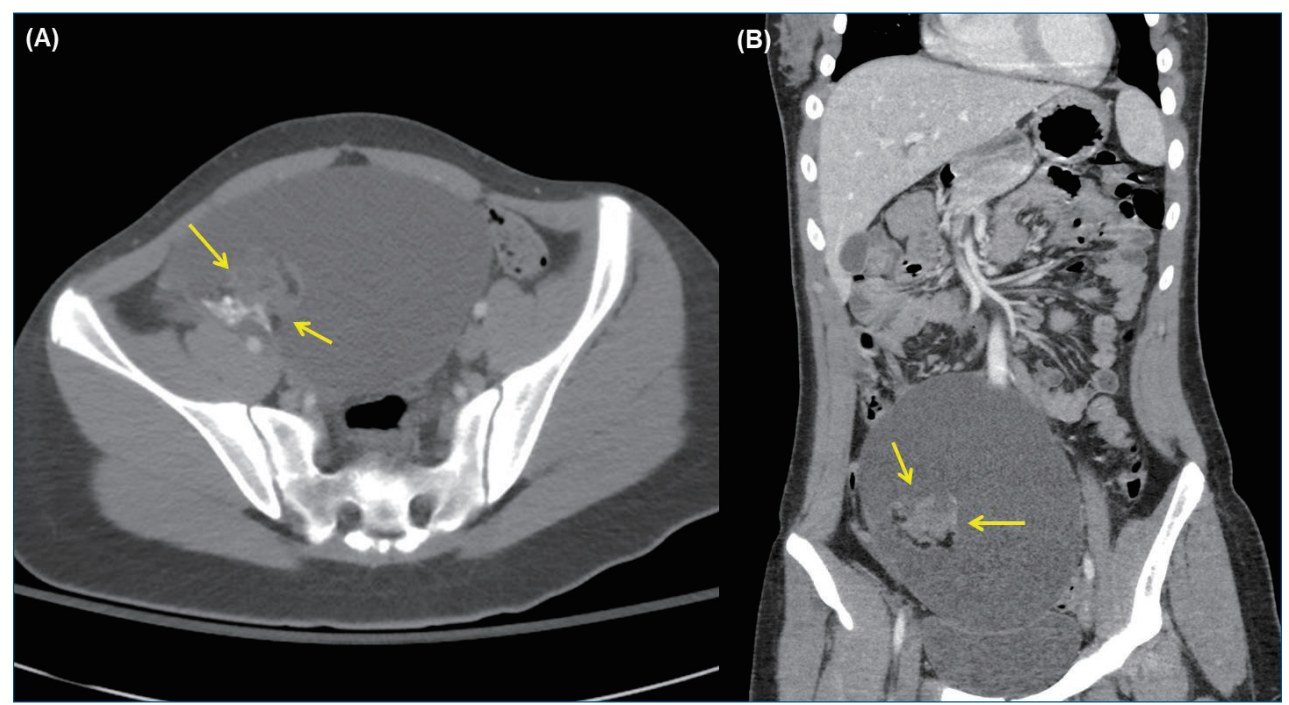

Fig. 1. Pelvic computed tomography scans of the axial (A) and coronal (B) views showing a cystic pelvic mass (arrows) with complex components measuring $3.8 \mathrm{~cm} \times 4 \mathrm{~cm} \times 3.6 \mathrm{~cm}$ along the right wall that contains soft tissue fat and calcium, findings that are most consistent with an ovarian dermoid cyst or teratoma.

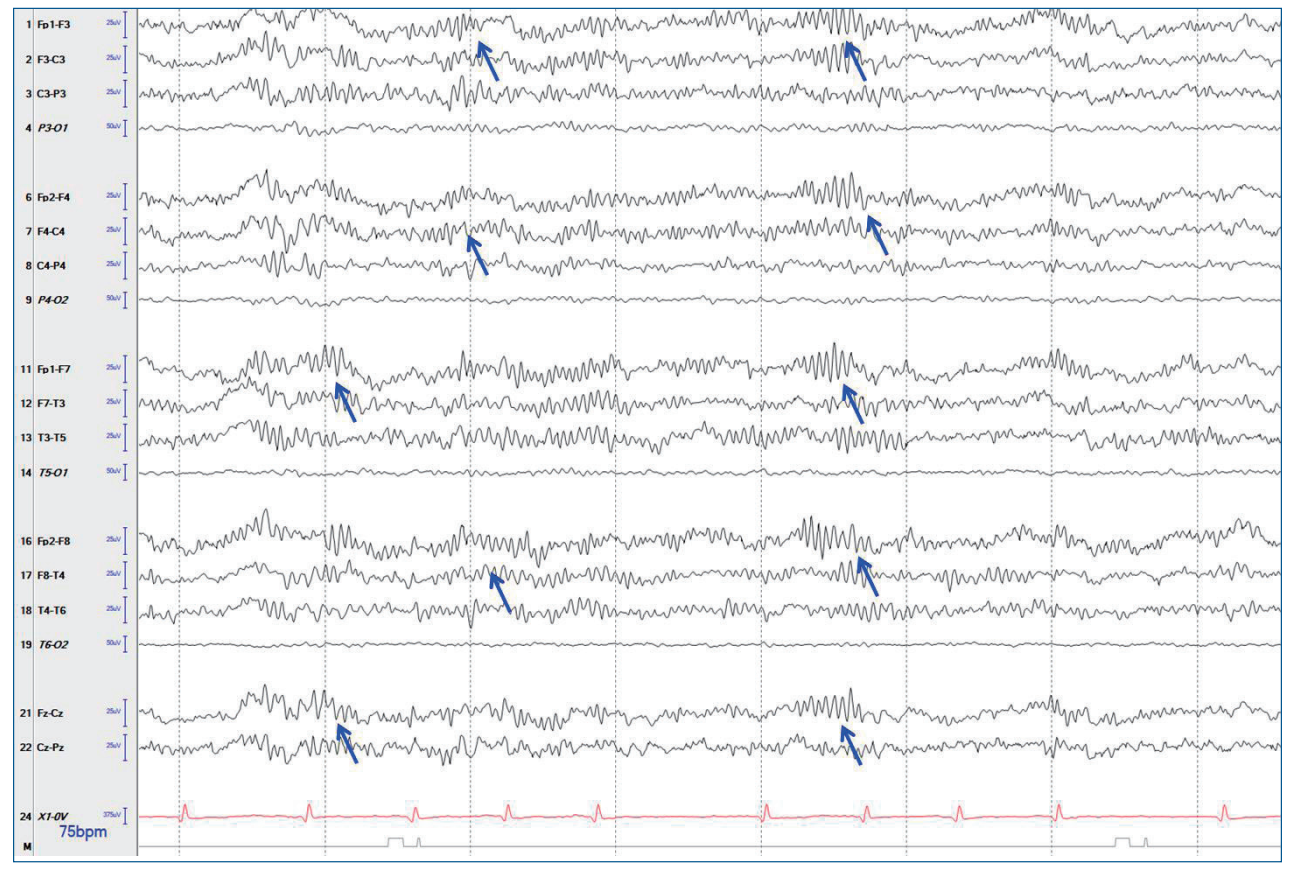

Fig. 2. Electroencephalogram (EEG) performed at 5 days after the initial presentation revealing diffuse delta activity with superimposed diffuse rhythmic beta frequency activity ("extreme delta brush" pattern, arrows). The EEG was acquired using the international 10-20 system of electrode placement with longitudinal bipolar montage, which was designed for 18 channels: Fp1-F3, F3-C3, C3-P3, P3-01, Fp2-F4, F4-C4, C4-P4, P4-02, Fp1-F7, F7-T3, T3-T5, T5-01, Fp2-F8, F8-T4, T4-T6, T6-O2, Fz-Cz, and Cz-Pz. 
varicella zoster virus, and Cryptococcus. Systemic infectious and rheumatologic evaluations were also negative. Pelvic computed tomography of a palpable mass found on an abdominal exam revealed a large cystic mass with a complex component (3.8 $\mathrm{cm} \times 4.9 \mathrm{~cm} \times 3.6 \mathrm{~cm}$ ) in her right ovary, which was most likely a dermoid cyst or teratoma (Fig. 1). She underwent surgical resection of the ovarian tumor. Twenty-four-hour video/EEG monitoring revealed continuous diffuse background slowing and extreme delta brushes (Fig. 2), suggesting diffuse encephalopathy related to anti-NMDA receptor (NMDAR) encephalitis. No AED was administered. The antibody and NMDAR test results were positive in the CSF (1:40 titer) but negative in the serum. Anti-voltage-gated potassium channel (VGKC), anti-glutamic acid decarboxylase (GAD), antiperoxidase, antithyroglobulin, and paraneoplastic antibodies tested negative in the CSF and serum.

The patient was administered high-dose intravenous (IV) methylprednisolone $20 \mathrm{mg} / \mathrm{kg}$ daily for 5 days and IV immunoglobulin G (IVIG) $400 \mathrm{mg} / \mathrm{kg}$ daily for 5 days. Her aggressive activity, ataxic gait, and dysarthria symptoms continued to wax and wane since the first-line immune therapy. No significant improvement was observed on video/EEG monitoring. She was treated with IV rituximab (a monoclonal anti-CD20 antibody, $375 \mathrm{mg} / \mathrm{m}^{2} / \mathrm{wk}$ ) as the second-line immune therapy. Her neurological symptoms and behavioral abnormalities improved significantly after IV rituximab therapy, while repeat MRI showed negative results; repeat CSF examination showed decreased anti-NMDAR antibody titer (1:20). Video/EEG findings became normal. She had no significant complications such as a serious infection or viral reactivation during the rituximab treatment. Final pathology of the ovarian mass showed a right cystic teratoma. She was discharged on day 19 with significant improvement in neurologic status and planned to continue active rehabilitation treatment. Presently, she is no longer taking antihypertensive drugs. At the 12-month follow-up, she reported continued improvement in her neurologic symptoms and a stable mood.

Table 1. Autoimmune or paraneoplastic encephalitis with antibodies against neuron-specific antigens

\begin{tabular}{|c|c|c|}
\hline Targeting antigen & Clinical features or main syndrome & Tumor associations \\
\hline \multicolumn{3}{|c|}{ Intracellular cytoplasmic antigens } \\
\hline ANNA-1 (anti-Hu) & $\begin{array}{l}\text { Encephalomyelitis (cortical, LE, BSE), cerebellar degeneration, sensory } \\
\text { neuropathy, autonomic dysfunction }\end{array}$ & SCLC, other \\
\hline ANNA-2 (anti-Ri) & Cerebellar degeneration, BSE, opsoclonus-myoclonus & Breast, gynecologic, SCLC \\
\hline Anti-Ma (Ma1, Ma2) & LE, BSE, peripheral neuropathy, hypothalamic dysfunction & $\begin{array}{l}\text { Testicular cancer, lung cancer, other solid } \\
\text { tumors }\end{array}$ \\
\hline Anti-CRMP-5 & $\begin{array}{l}\text { Encephalitis, optic neuritis, retinitis, myelopathy, Lambert-Eaton } \\
\text { myasthenic syndrome }\end{array}$ & SCLC, breast cancer \\
\hline Anti-amphiphysin & Stiff-person syndrome, LE & Breast, lung cancer \\
\hline Anti-GAD65 & $\begin{array}{l}\text { LE, stiff-person syndrome, BSE, cerebellar ataxia, diabetes, ocular } \\
\text { movement disorder }\end{array}$ & Thymoma, renal cell, breast, or colon cancer \\
\hline \multicolumn{3}{|l|}{ Plasma membrane antigens } \\
\hline \multicolumn{3}{|l|}{ Synaptic receptors } \\
\hline NMDAR & $\begin{array}{l}\text { Psychosis, insomnia, seizures, behavior change, memory impairment, } \\
\text { autonomic dysfunction, catatonia, coma }\end{array}$ & Age-dependent ovarian teratoma \\
\hline GABAA receptor & Rapid progressive encephalopathy, refractory seizures, status epilepticus & $40 \%$ (thymoma) \\
\hline GABAB receptor & Prominent seizures, LE, cerebellar ataxia, opsoclonus-myoclonus & $50 \%$ (mostly SCLC) \\
\hline AMPA receptor & LE, psychiatric disturbances, memory loss & Thymoma, SCLC \\
\hline GlyR & $\begin{array}{l}\text { LE with status epilepticus, encephalomyelitis, muscle spasm, rigidity, } \\
\text { hyperekplexia }\end{array}$ & Past history of cancer, concurrent cancer \\
\hline mGluR5 & Confusion, encephalitis (Ophelia syndrome) & Hodgkin lymphoma \\
\hline mGluR1 & Cerebellar ataxia & Hodgkin lymphoma \\
\hline Dopamine-2 receptor & Basal ganglia encephalitis & No \\
\hline \multicolumn{3}{|c|}{ Ion channels and/or cell surface } \\
\hline LGI1 & LE, seizures, FBDS, rapidly progressive cognitive decline, hyponatremia & Thymoma (5\%-10\%), hyponatremia (60\%) \\
\hline Caspr2 & $\begin{array}{l}\text { Insomnia, Morvan syndrome, peripheral nerve hyperexcitability (neuro- } \\
\text { myotonia), LE, neuropathic pain, autonomic dysfunction, ataxia }\end{array}$ & Thymoma, variable solid tumors \\
\hline DPPX & $\begin{array}{l}\text { Encephalopathy, hyperexcitability, myoclonus, tremor, diarrhea, weight } \\
\text { loss }\end{array}$ & Lymphoma \\
\hline MOG & Acute disseminated encephalomyelitis & No \\
\hline Aquaporin 4 & Encephalitis, neuromyelitis optica & No \\
\hline GQ1b & BSE & No \\
\hline
\end{tabular}

AMPA, $\alpha$-amino-3-hydroxy-5-methyl-4-isoxazolepropionic acid; ANNA-1, antineuronal nuclear antibody type 1; LE, limbic encephalitis; BSE, brainstem encephalitis; SCLC, small-cell lung cancer; CRMP-5, collapsing response mediator protein-5; GAD, glutamic acid decarboxylase; NMDA, N-methyl-D-aspartate; GABA, $\gamma$-aminobutyric acid; GlyR, glycine receptor; mGluR, the metabotropic glutamate receptor; LGI1, leucine-rich glioma inactivated-1; FBDS, faciobrachial dystonic seizures; Caspr2, contactin-associated protein-like 2; DPPX, dipeptidyl-peptidase-like protein; MOG, myelin oligodendrocyte glycoprotein. 


\section{Neuron-specific autoantibodies}

As with other autoimmune disorders affecting the nervous system, 3 types of autoantibodies have been recognized according to antigen location: (1) those targeting intracellular proteins; (2) those targeting plasma membrane proteins including synaptic receptors; and (3) those targeting ion channels and/or other cell surface proteins (Table 1$).^{5-7)}$

Antibodies against intracellular antigens are commonly detected in patients with cancer, mainly small-cell carcinoma, and are typical in adults; they are defined as paraneoplastic antibodies but are unlikely to cause neurological manifestations alone. ${ }^{8)}$ The most important antibodies included in this group are against $\mathrm{Ma}$, $\mathrm{Hu}$ (also defined as type 1 antineuronal nuclear autoantibody), and $\left.\mathrm{GAD} .{ }^{6}\right)$ The intracellular antigens can be exposed to autoantibodies within the synaptic cleft, ${ }^{9}$ a cytotoxic T-cellmediated immune response against neurons, is more likely to be the main mechanism leading to specific damage. In all cases, the response to immunosuppressive therapy is poor.

The pathogenesis of neuronal damage and patterns of response to therapy in autoimmune encephalitis, in which the immune response targets proteins that are part of synaptic receptors, ion channels, or cell surface, is different. Unlike patients with antibodies to intracellular targets, those with antibodies against plasma membrane protein or ion channels respond remarkably well to immunotherapy. These antibodies, disrupting the target antigen, are the true cause of damage. ${ }^{10)}$ The same phenomenon may occur when drugs or genetic mutations cause disruption of the target antigen. Synaptic receptor antigens involved in autoimmune encephalitis include NMDA, $\gamma$-aminobutyric acids A and $B$ receptors $\left(G_{A B A_{A}} R, G A B A_{B} R\right), \alpha$-amino-3-hydroxy-5methyl-4-isoxazolepropionic acid (AMPA), metabotropic glutamate receptor 5 , and the dopamine 2 receptor. ${ }^{2,6,10)}$ Antigens that are part of ion channels or the cell surface include leucine-rich glioma inactivated-1 (LGI1), contactin-associated protein-like 2 (Caspr2), dipeptidyl-peptidase-like protein 6 (DPPX), myelin oligodendrocyte glycoprotein, aquaporin 4 , and ganglioside

Table 2, Diagnostic criteria for possible autoimmune encephalitis ${ }^{6}$

All 3 of the following criteria must be met:
1. Subacute onset (rapid progression of $<3$ months) of working memory
deficits (short-term memory loss), altered mental status ${ }^{\mathrm{a}}$, or psychi-
atric symptoms
2. At least one of the following:
New focal CNS findings
Seizures not explained by a previously known seizure disorder
CSF pleocytosis (>5 WBC/ $/ \mathrm{mm}^{3}$ )
MRI features suggestive of encephalitis ${ }^{\text {b) }}$
3. Reasonable exclusion of alternative causes

3. Reasonable exclusion of alternative causes

CNS, central nervous system; CSF, cerebrospinal fluid; WBC, white blood cell; $\mathrm{MRI}$, magnetic resonance imaging.

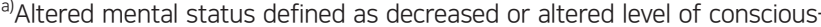
ness, lethargy, or personality changes. ${ }^{b}$ MRI hyperintense signal on T2weighted image/fluid-attenuated inversion recovery highly restricted to one or both medical temporal lobes, or in multifocal areas involving grey and/or white matter compatible with demyelination of inflammation.
GQ1b. 2,6,10)

However, identifying antibodies involved in different autoimmune encephalitis is difficult, as it requires complicated laboratory methods that are not readily available in many institutions. Therefore, an autoimmune encephalitis diagnosis should be suspected following a combination of proper signs and symp. toms. The initial assessment should include a neurological evaluation and standard diagnostic tests including MRI and CSF study. The diagnosis of autoimmune encephalitis should be considered at all ages when all 3 of the following criteria have been met (Table 2): (1) subacute onset of working memory deficits (shortterm memory loss), altered mental status (decreased or altered level of consciousness, lethargy, or personality change), or psy. chiatric symptoms; (2) at least one of the following: new focal CNS findings; seizures not explained by a previously known seizure disorder; CSF pleocytosis with a WBC count $>5 / \mathrm{mm}^{3}$; MRI features suggestive of encephalitis (hyperintense signal highly restricted to one or both medial temporal lobes (as in limbic encephalitis [LE]), or in multifocal areas involving grey matter, white matter, or both compatible with demyelination or inflammation); and (3) reasonable exclusion of alternative causes. ${ }^{6}$

\section{Specific autoimmune encephalitis syndrome}

Although clinical manifestations of autoimmune encephalitis can vary significantly, different autoantibodies can be associated with similar phenotypes, certain clinical features suggest a specific type of autoimmune encephalitis, such as facio-brachial dystonic seizures (FBDS) and memory impairment with antiLGI1 encephalitis; encephalopathy, insomnia, ataxia, peripheral nerve hyperexcitability, and neuropathic pain with anti-Caspr2 encephalitis; refractory SE with anti-GABAA R encephalitis; and myoclonus, tremors, hyperexcitability, and severe diarrhea with anti-DPPX encephalitis (Table 1). Seizures and movement disorders along with psychosis, confusion, or additional behavioral changes are the most relevant initial manifestations in all age groups.

\section{Anti-NMDAR encephalitis}

Anti-NMDAR encephalitis is the most common and best characterized autoimmune encephalitis syndrome. ${ }^{11,12)}$ Although this encephalitis type can affect people of any age, it is predominant in young women and children. In many patients, both neurological signs and symptoms are associated with those of a tumor, most commonly an ovarian teratoma, although testicular immature teratoma and lymphoma have been also described. ${ }^{11-13)}$

NMDARs are ligand-gated ion channels that mediate excitatory neurotransmission, are included in a group of ionotropic glutamate receptors with tetrameric complexes of large transmembrane subunits. ${ }^{14)}$ Three different NMDAR subunits have been identified: NR1, NR2, and NR3. The simultaneous binding of glycine and glutamate activates NMDAR, which results 
in cation (mainly $\mathrm{Ca}^{2+}$ ) influx into the cell provided that sufficiently strong depolarization is present. NMDAR signaling is critical for activity-dependent synaptic plasticity and long-term hippocampal potentiation. ${ }^{15)}$ Moreover, NMDARs modulate excitotoxicity, as excessive cation influx can cause neuronal death. ${ }^{16)}$ In anti-NMDAR encephalitis, the autoimmune process causes a selective and reversible decrease in NMDAR surface density and synaptic localization, leading to disruption of the synaptic structure and function, strictly dependent on the patient's antibody titer. ${ }^{17)}$ An autoimmune response to the NR1 subunit of NMDAR has been shown in children to be triggered by various infectious agents, and different reports have suggested that anti-NMDAR encephalitis may be associated with HSV, Mycoplasma pneumoniae, measles virus, mumps, influenza A/ H1N1 infection, group A hemolytic Streptococcus, and Toxoplasmagondii infection. ${ }^{18,19)}$

In a multicenter observational study of 577 patients, the disease predominantly affected young people with a female sex predominance of $4: 1$. This predominance was less evident in children aged $<12$ years and adults aged $>45$ years. ${ }^{11)}$ Many patients present with prodromal headache, fever, or a viral-like process, followed in a few days by multistage progression of characteristic symptoms: prominent psychiatric manifestations (anxiety, agitation, bizarre behavior, hallucinations, delusions, disorganized thinking), insomnia, memory deficits, seizures, cognitive impairment, movement disorders (dyskinesias, choreoathetoid, dystonia, tremor, ataxia, rigidity, opisthotonic postures), language dysfunction, autonomic instability (hyperthermia, blood pressure fluctuations, tachycardia, bradycardia, and sometimes hypoventilation requiring mechanical ventilation). ${ }^{20}$ Clinical manifestations differ between adults and children. First, the association with a tumor is less common in children. Approximately $50 \%$ of females aged $>18$ years have uni- or bilateral ovarian teratomas versus $<9 \%$ of girls aged $<14$ years. ${ }^{11)}$ In teenagers and adults, neuropsychiatric manifestations (psychosis, delusion, agitation, aggression, or catatonia), memory loss, and hallucinations are the rule at onset. Contrastingly, only $60 \%$ of children present exclusively with psychiatric symptoms, whereas movement disorders and seizures are significantly more common. Seizures can occur at any time but tend to strike earlier in males, being general or focal. ${ }^{21)}$ Regardless of patient age and presentation, the clinical features at 3-4 weeks after symptom onset are similar in most cases within several categories.

CSF pleocytosis is significantly less common in children than in adults (43\% vs. $63 \%$, respectively; $P=0.0163){ }^{20)}$ Almost all patients have abnormal EEG findings at disease onset and peak. Diffuse slowing is the most common feature in afflicted children and adults. ${ }^{22)}$ Moreover, at the peak stage, focal slowing and epileptiform discharges, polymorphic delta rhythm, diffuse fast activities, and extreme delta brush can also be detected. ${ }^{23)}$ MRI findings are often normal or show medial temporal and frontal hyperintensity on T2-weighted images and leptomeningeal contrast enhancement. ${ }^{24)}$ Subcortical white matter changes seem as common as those of cortical grey matter. Positron emission tomography (PET) can show CNS abnormalities in cases with normal MRI findings. ${ }^{24)}$ However, very few studies have used this diagnostic tool in patients with anti-NMDAR encephalitis; thus, further research is needed to evaluate the role of PET in diagnosing this syndrome. An anti-NMDAR encephalitis diagnosis is confirmed by the detection of immunoglobulin $\mathrm{G}$ (IgG) antibodies to the GluN1 (also known as NR1) subunit of NMDAR in the serum or CSF. ${ }^{25)}$ CSF IgG antibody testing is highly sensitive and specific for anti-NMDAR encephalitis; false-positive and -negative results may occur when only serum is tested. While waiting for confirmatory IgG anti-Glu1 antibody results, we should assess a patient with promptly progressive encephalopathy as having probable anti-NMDAR encephalitis if they fulfill the criteria shown in Table 3. ${ }^{6}$

Treatment options include immunosuppression and tumor resection when indicated. ${ }^{11,26)}$ In the absence of prospective and randomized data, treatment decisions should be individualized and consider patient age, tumor presence or absence, and symptom severity. Based on the observational studies reviewed below and our clinical experience, we recommend first-line immunotherapy involving IV methylprednisolone (e.g., 1 g daily for 5 days in an adult), IVIG (e.g., $400 \mathrm{mg} / \mathrm{kg} /$ day for 5 days)

\title{
Table 3. Diagnostic criteria for probable anti-NMDA receptor encephalitis
}

\author{
All 3 criteria must be met: \\ 1. Rapid onset ( $<3$ months) of at least 4 of the 6 following major groups of symptoms ${ }^{\text {a) }}$ : \\ Abnormal (psychiatric) behavior or cognitive dysfunction \\ Speech dysfunction \\ Seizures \\ Movement disorder, dyskinesias, or rigidity/abnormal postures \\ Decreased level of consciousness \\ Autonomic dysfunction or central hypoventilation \\ 2. At least one of the following laboratory results: \\ Abnormal EEG (focal or diffuse slow or disorganized activity, epileptic activity, or extreme delta brush) \\ CSF with pleocytosis or oligoclonal bands \\ 3. Reasonable exclusion of other disorders \\ NMDA, N-methyl-D-aspartate; EEG, electroencephalogram; CSF, cerebrospinal fluid. \\ ${ }^{a}$ In the presence of a systemic teratoma, the diagnosis can be made in the presence of 3 groups of symptoms.
}


or plasma exchange alone or combined with tumor removal, when appropriate. It is unknown whether IVIG and plasma exchange have similar efficacies. If there is no evidence of clinical improvement with initial therapies, we progress to second-line therapies including rituximab (either $375 \mathrm{mg} / \mathrm{m}^{2}$ weekly for 4 weeks, or $1 \mathrm{~g}$ twice 2 weeks apart), and cyclophosphamide (750 mg/m² monthly for 4-6 months depending on the results) alone or combined. These second-line drugs are recommended if no sustained improvement occurs within 4 weeks of immunotherapy initiation or tumor removal. ${ }^{11)}$ Factors associated with better prognosis included no need for intensive care unit admission $(P<0.0001)$, early treatment $(P=0.009)$, and low disease severity within 4 weeks from onset $(P=0.011){ }^{5}$ )

\section{VGKC-positive group of autoimmune encephalitis}

These diseases start as LE with cognitive impairment, seizures, and variable neuropsychiatric symptoms.

\section{1) Anti-LGI1 encephalitis}

The associated antibodies target the secreted neuronal protein LGI1, functioning as a ligand for 2 epilepsy-related proteins, ADAM22 and ADAM23, that are essential for inhibitory signal transmission from the presynaptic potassium channel to the postsynaptic AMPA receptors. ${ }^{27,28)}$ Antibody binding to LGI1 disrupts pre- and postsynaptic LGI1 signaling, resulting in neuronal hyperexcitability. ${ }^{29)}$

Anti-LGI1 encephalitis occurs in approximately 60-year-old men presenting with LE. The clinical features are homogeneous and include seizures and subacute progressive memory alterations; behavior and space orientation abnormalities are the most common signs. In some cases, seizures precede the cognitive decline. ${ }^{30)}$ The most characteristic seizures, detectable in approximately 50\% of cases, are FBDS. FBDS, considered pathognomonic of anti-LGI1 encephalitis, are involuntary unilateral contractions of the face and arm (or leg) that last for $<3$ seconds and occur up to 100 times a day. Generalized tonic-clonic seizures can also be seen in the late disease stage. Memory and cognitive deficits may be preceded by short FBDS. ${ }^{31,32)}$ Patients can develop hyponatremia (nearly 60\%) and rapid eye movement sleep behavior disorder. EEG can reveal the multifocal interictal epileptiform discharges and slow waves. MRI usually reveals findings typical of LE (e.g., medial temporal lobe hyperintensity), while CSF findings are often normal or include only oligoclonal bands. ${ }^{33,34)}$ Approximately 5\%-11\% of cases are associated with cancer; the most common associated tumor is thymoma. The association with other tumors may be coincidental. ${ }^{30)}$

Treatment with glucocorticoids, IVIG, mycophenolate mofetil, and/or plasma exchange results in significant improvement in 70\%-80\% of patients. ${ }^{31)}$ The early immunotherapy initiation in patients with FBDS may prevent cognitive impairment and improve long-term outcomes. ${ }^{32)}$ However, experience with rituximab as an add-on therapy is limited. ${ }^{35)}$ Relapses occur in up to one-third of patients and are associated with worse outcomes. Despite substantial recovery, cognitive deficits and disability persist in many patients along with evidence of hippocampal atrophy on MRI. ${ }^{36)}$

\section{2) Anti-Caspr2-associated encephalitis}

The target antigen is Caspr2, an adhesion molecule expressed in the CNS and peripheral nervous system (PNS) that is essential for maintaining normal function of the VGKC. ${ }^{37)}$ AntiCaspr2-associated encephalitis can manifest as LE: Morvan syndrome (neuromyotonia, memory loss and confusion, sleep disturbances, autonomic dysfunction), and in few patients, as isolated neuromyotonia. ${ }^{34,37,38)}$

This disorder predominantly affects older men (median age, 65 years), although rare pediatric cases have been described. ${ }^{38,39)}$ The presentation and disease course are slower than those of other autoimmune encephalitis syndromes. Although the clinical syndrome is less homogeneous than anti-LGI1 encephalitis, almost $80 \%$ of patients develop $\geq 3$ of the core symptoms ("strongly supported diagnostic symptoms"): encephalopathy (cognitive decline/seizures), cerebellar symptoms, PNS hyperexcitability, autonomic dysfunction, insomnia, neuropathic pain, and weight loss, symptoms that might progress very slowly, mimicking dementia. ${ }^{39,40)}$ Due to PNS involvement, muscular pain, fasciculations, and cramps are present in many patients. The disorder is usually not associated with cancer. A tumor, usually thymoma, may be revealed in up to $32 \%$ of cases. ${ }^{41)}$ Patients with tumors (commonly thymoma) are more likely to develop Morvan syndrome than those with isolated central or peripheral symptoms. CSF pleocytosis is seen in 30\%-40\% of cases. MRI findings are normal in most patients or consist of bilateral T2 hyperintensity of the medial temporal lobes.

Tumor removal is crucial for improvement and cure. Immunotherapy seems effective in patients without malignancy. However, relapse affects approximately 25\%, occurring even several years after the initial episode and involving other areas of the CNS. ${ }^{39)}$ The presence of autoantibodies directed against the synaptic protein LGI1 and adhesion molecule Caspr2 has not been found in children with acute encephalitis; thus, the role of these autoantibodies in the pediatric population is still debated.

3) VGKC-positive encephalitis in the absence of anti-LGI1 and Caspr2 antibodies

The clinical features of antibodies against VGKC in patients lacking anti-LGI1 and anti-Caspr2 antibodies has not been precisely defined. These patients have a heterogeneous clinical spectrum, including pain syndrome, LE, epilepsy, polyneuropathy, and cramp fasciculation syndromes, all associated with the demonstration of anti-VGKC autoantibodies. ${ }^{42}$ An acute and isolated psychotic manifestation of anti-VGKC encephalitis was recently reported in a 16-year-old girl, but further studies are required to recognize potential new antibodies against neuronal structures. $^{43)}$

\section{Anti-GABAR encephalitis}

Neurotransmitter $\gamma$-aminobutyric acid (GABA) is the chief 
inhibitory neurotransmitter in the vertebrate CNS. GABAA R are ligand-gated ion channels that allow the influx of $\mathrm{Cl}^{-}$and fast synaptic inhibition; metabotropic GABAB $\mathrm{R}$ are expressed in the CNS and PNS: This effect is primarily inhibitory via the inhibition of presynaptic voltage-gated $\mathrm{Ca}^{2+}$ channels, activation of postsynaptic $\mathrm{K}^{+}$channels, and inhibition of adenylyl cyclase. ${ }^{44)}$

Patients with anti-GABA $\mathrm{R}$ encephalitis develop rapidly progressive encephalitis with refractory seizures, $\mathrm{SE}$, and/or epilepsia partialis continua. ${ }^{45)}$ Nearly $50 \%$ of reported cases involve children. ${ }^{46)} \mathrm{CSF}$ often shows lymphocytic pleocytosis with increased protein concentrations. Unlike other causes of autoimmune encephalitis, the MRI in anti-GABA $\mathrm{A}$ encephalitis often shows multifocal cortical/subcortical and widespread abnormal sig. naling on fluid-attenuated inversion recovery (FLAIR) and T2weighted scans. ${ }^{46)}$ Tumors (usually thymoma) occur in $40 \%$ of patients, almost all of whom are adults. ${ }^{45,46)}$ In children, anti$\mathrm{GABA}_{\mathrm{A}} \mathrm{R}$ encephalitis may progress as a postviral encephalitis and coexist with NMDAR antibodies. A case of a previously healthy child presenting with catatonia and encephalopathy without seizures due to antibodies against $\mathrm{GABA}_{\mathrm{A}} \mathrm{R}$ was recently reported. ${ }^{47)}$ Patients respond to immunotherapy but often require pharmacologic-induced coma for prolonged seizures.

Encephalitis due to antibodies against the $\mathrm{GABA}_{B} \mathrm{R}$ was described primarily in adults presenting with LE, including seiures, SE, ataxia, or opsoclonus-myoclonus. ${ }^{48)}$ One pediatric case was characterized by encephalopathy, refractory seizures, and a mixed movement disorder (opsoclonus, ataxia, and chorea) ${ }^{49)}$ Approximately 50\% of cases are paraneoplastic and almost always associated with small-cell lung cancer. ${ }^{48)}$ MRI reveals FLAIR and T2 hyperintensity consistent with LE in approximately 50\%; over one-half of patients have CSF pleocytosis and/or elevated protein levels. ${ }^{48)}$ The response to immunosuppressive therapies was good in tumor-free patients.

\section{Anti-AMPA receptor encephalitis}

Encephalitis associated with antibodies against the AMPA receptor predominantly affects females, with a median age of onset of 50-60 years. ${ }^{50,51)}$ In a case series of 22 patients with antiAMPA receptor antibodies, LE with or without seizures was the most common clinical presentation (55\%); other manifestations included limbic dysfunction along with diffuse encephalopathy (36\%), motor deficits followed by LE (one patient), and psychosis with bipolar features (one patient). ${ }^{51)}$ An underlying tumor is detected in approximately two-thirds of the patients, most commonly in the lung, thymus, or breast. ${ }^{50,51)}$ CSF lymphocytic pleocytosis has been described in 50\% to 90\% (WBC count, $\left.5-164 / \mathrm{mm}^{3}\right){ }^{50,51)}$ MRI findings are usually abnormal, with FLAIR signal abnormalities in the medial temporal lobes. In one study, 9 of 10 patients responded to the tumor resection and/ or immunotherapy, but consequent relapses occurred in 5 (in the absence of tumor recurrence) and were associated with an incomplete treatment response and death of SE in one patient. ${ }^{50)}$

\section{Anti-glycine receptor encephalopathy}

Glycine receptors (GlyRs) are ligand-gated chloride channels that mediate fast inhibitory transmission, leading to neural hyperpolarization through a selective influx of chloride. GlyRs are essential for muscle tone, coordination, respiratory rhythm, and sensory processing. ${ }^{52)}$ The genetic modification of GlyRs is associated with hypertonia, hyperekplexia, development delay, apnea, and stiff-person syndrome in some patients. ${ }^{53)}$ Patients with anti-GlyR antibodies often have progressive encephalomyelitis with rigidity and myoclonus, although severity and clinical expressions vary. ${ }^{54)} \mathrm{A}$ few patients have been reported with limbic or other encephalopathy without brainstem or spinal cord features. In one study of 45 patients, 5 had a past history and successful treatment of a tumor, while 4 were concurrently diagnosed with tumor and neurologic disease. ${ }^{55)}$ They reported that, at onset, spasms, which were often painful, and stiffness/ rigidity of the neck, trunk, or limb muscles were demonstrated in $69 \%$ of patients, whereas diplopia, ptosis, and nystagmus were found in $40 \%$ of patients. Finally, cognitive impairment was observed in 29\%, while seizures occurred in $13 \%$.

\section{Anti-GAD encephalitis}

GAD is an intracellular enzyme that is found in neural and pancreatic cells. ${ }^{56}$ ) Two isoforms of GAD (GAD 65 and GAD 67) have been identified; both can be detected in the CNS, where they catalyze GABA (the major inhibitory neurotransmitter) synthesis, whereas pancreatic islet cells contain only GAD ${ }^{65.56}$ ) Antibodies against $\mathrm{GAD}$ are associated with both type 1 diabetes and several neurological manifestations, including LE, stiffperson syndrome, cerebellar ataxia, ocular movement disorders, and refractory epilepsy. ${ }^{56}$ Very few cases have been found in children. In particular, LE with anti-GAD 65 antibodies is characterized by rapidly progressive short-term memory loss, psychiatric symptoms, and seizures; it commonly has a chronic course with poor response to immunotherapy and AEDs. ${ }^{57,58)}$ Stiff-person syndrome with abnormal postures (often hunched over and stiffened) are characteristics of this disorder. Malig. nancy (thymoma or small-cell lung carcinoma) is comorbid with approximately $25 \%$ of cases of anti-GAD 65 encephalitis. ${ }^{6}$ Recently, a 9-year-old girl was reported with refractory seizures and behavioral and severe fatal autonomic dysfunction, including arrhythmia, bradycardia/tachycardia, hypotension/hypertension, hypothermia/hyperthermia, and hyperhidrosis, demonstrating the possibility of extralimbic brain involvement in children with anti-GAD 65 antibodies. ${ }^{59)}$

\section{Diagnostic approach}

In the appropriate clinical presentation, detecting specific autoantibodies confirms the autoimmune encephalitis diagnosis. However, not all patients with paraneoplastic or autoimmune encephalitis have antibodies, and the absence of antibodies does not rule out an autoimmune mechanism. For patients without 


\begin{tabular}{|c|c|}
\hline Etiology & Disorders \\
\hline Infectious etiologies & $\begin{array}{l}\text { Viral encephalitis (HSV, HHV6, VZV, EBV, CMV, HIV, enterovirus, arbovirus); bacterial encephalitis (Listeria, } \\
\text { Bartonella, Mycoplasma, Rickettsia); spirochetal encephalitis; fungal infection; tuberculosis; Creutzfeldt- } \\
\text { Jakob disease; Whipple disease }\end{array}$ \\
\hline Toxic-metabolic & $\begin{array}{l}\text { Drug ingestion (alcohol, ketamine, phencyclidine, organophosphates); carbon monoxide; Wernicke } \\
\text { encephalopathy; neuroleptic malignant syndrome }\end{array}$ \\
\hline Vascular disorders & $\begin{array}{l}\text { Reversible posterior leukoencephalopathy syndrome, primary or secondary angiitis, Behcet disease, Susac } \\
\text { syndrome (autoimmune vasculopathy) }\end{array}$ \\
\hline Neoplastic disorders & Leptomeningeal metastases, diffuse glioma, primary or secondary CNS lymphoma \\
\hline Demyelinating or inflammatory disorders & Multiple sclerosis, neuromyelitis optica, acute disseminated encephalomyelitis, neurosarcoidosis \\
\hline Neurodegenerative dementias & Alzheimer disease, frontotemporal dementia, dementia with Lewy bodies, vascular cognitive impairment \\
\hline Psychiatric disease & Schizophrenia, bipolar disorder, conversion disorder, substance abuse \\
\hline Inherited and metabolic disorders & Mitochondrial cytopathies \\
\hline
\end{tabular}

antibodies, the suspicion of an autoimmune or paraneoplastic etiology is based on the clinical history of a subacute progressive neurologic course, cancer risk factors, and supportive laboratory and radiologic results.

The differential diagnosis of autoimmune or paraneoplastic encephalitis includes various broad categories, such as infection, toxic and metabolic disturbances, vascular disorders, neoplastic disorders, demyelinating and inflammatory disorders, psychiatric diseases, neurodegenerative dementias, and rare heritable or metabolic disorders (Table 4). ${ }^{25,60)}$ Patients with suspected autoimmune encephalitis (Table 2) should undergo neuroimaging, EEG, lumbar puncture, and serologic testing for appropriate biomarkers to confirm the diagnosis and exclude alternative etiologies.

Distinctive MRI findings of autoimmune encephalitis include signal hyperintensities on FLAIR or T2-weighted images in affected brain regions (e.g., medial temporal lobes and/or brainstem, subcortical regions, and cerebellum). Contrast enhancement is variable. ${ }^{45)}$ EEG should be performed to exclude nonconvulsive seizures. Nonspecific abnormal findings are common and include focal or generalized slowing, epileptiform discharges, and periodic lateralized epileptiform discharges. ${ }^{61)}$ Approximately one-third of patients with NMDAR encephalitis have the finding of extreme delta brush, considered a characteristic of the disorder. ${ }^{23)}$ The mechanism underlying the delta brush remains unclear. As a cellular mechanism, the modulation of NMDAR-mediated currents was proposed by altering rhythmic neuronal activity and leading to this pattern. ${ }^{23)}$ Furthermore, delta brush was recently found among intracranial EEG ictal-onset patterns related to focal cortical dysplasia in a series including hippocampal sclerosis with different epileptogenic lesions. Therefore, delta brush is not pathognomonic for NMDAR encephalitis. But its presence still can guide early recognition and prompt immunotherapy in NMDAR encephalitis, especially in patients with proper clinical features when other possible etiologies are excluded. ${ }^{62)}$ A CSF examination should be performed to exclude viral infection and leptomeningeal metastasis. Patients with autoimmune encephalitis may or may not have abnormal CSF findings. Abnormalities include modestly elevated protein $(<100 \mathrm{mg} / \mathrm{dL})$, mild to mo- derate lymphocytic pleocytosis, an elevated IgG index, and/or the presence of oligoclonal bands. ${ }^{61,63)}$ However, these findings are variable. The absence of inflammatory evidence on CSF and MRI may be common in older adults. ${ }^{64)}$ Screening for malignancy is mandatory in children to rule out a neoplastic syndrome.

\section{Therapeutic approach}

Immunosuppressive therapy should not be delayed until a cancer diagnosis confirmation or antibody results in patients with a typical paraneoplastic syndrome or autoimmune encephalitis provided that an underlying infectious etiology has been ruled out and there are no other contraindications. ${ }^{65)}$ There are no evidence-based treatment standards for autoimmune encephalitis, although many clinicians have become confident with the prompt use of high-dose methylprednisolone combined with IVIG. ${ }^{66)}$ Plasma exchange is also useful first-line acute treatment for critically ill patients or when IV steroid or IVIG is poorly tolerated. After an initial trial of therapy, patients should be reevaluated for clinical improvement. Rituximab or cyclophosphamide can be considered second-line agents when there is no or an incomplete response to first-line treatment. SE in a patient with anti-NMDAR antibodies or LE and LGI1 positivity may more rapidly escalate immunotherapy.

Other immunotherapies could be considered in post-infectious cases such as immunoadsorption (antibody-removing therapy) alternative to plasma exchange, which should be started as promptly as possible to warrant the best neurological outcomes, although several weeks or months might be required to show clear effectiveness. ${ }^{67)}$ More research on differential bloodbrain barrier permeability would resolve many questions about autoantibody entry and the pathogenesis of autoimmune encephalitis. There are no data about anticytokine therapies in young patients with refractory autoimmune encephalitis. Overall outcomes are usually good in younger patients with autoimmune encephalitis, although this depends on the underlying tumor and stage as well as the severity of the initial neurological symptoms. ${ }^{68)}$ The delay to diagnosis and treatment has been associated 
with worse prognosis and increased relapse rates. ${ }^{11)}$

\section{Conclusion}

The proper diagnosis and prompt management of autoimmune encephalitis require an organized approach. The evaluation should begin with a detailed history and physical examination to detect clues to specific causes. Ancillary MRI, EEG, and CSF testing may further support a diagnosis of encephalitis and exclude infectious causes. Antineuronal antibody tests are used to make the diagnosis, but a negative antibody test may not exclude autoimmune disorders indefinitely. Treatment for suspected autoimmune encephalitis could be considered prior to antibody test results are available since prompt treatment is associated with better outcomes.

\section{Conflicts of interest}

No potential conflict of interest relevant to this article was reported.

See the commentary "Autoimmune encephalitis and epilepsy: evolving definition and clinical spectrum" via https://doi.org/ 10.3345/cep.2020.00353.

\section{References}

1. Venkatesan A, Tunkel AR, Bloch KC, Lauring AS, Sejvar J, Bitnun A, et al. Case definitions, diagnostic algorithms, and priorities in encephalitis: consensus statement of the international encephalitis consortium. Clin Infect Dis 2013;57:1114-28.

2. Varley J, Taylor J, Irani SR. Autoantibody-mediated diseases of the CNS: structure, dysfunction and therapy. Neuropharmacology 2018;132:7182.

3. Quek AM, Britton JW, McKeon A, So E, Lennon VA, Shin C, et al. Autoimmune epilepsy: clinical characteristics and response to immunotherapy. Arch Neurol 2012;69:582-93.

4. Toledano M, Britton JW, McKeon A, Shin C, Lennon VA, Quek AM, et al. Utility of an immunotherapy trial in evaluating patients with presumed autoimmune epilepsy. Neurology 2014;82:1578-86.

5. Esposito S, Principi N, Calabresi P, Rigante D. An evolving redefinition of autoimmune encephalitis. Autoimmun Rev 2019;18:155-63.

6. Graus F, Titulaer MJ, Balu R, Benseler S, Bien CG, Cellucci T, et al. A clinical approach to diagnosis of autoimmune encephalitis. Lancet Neurol 2016;15:391-404.

7. Toledano M, Pittock SJ. Autoimmune epilepsy. Semin Neurol 2015;35: 245-58.

8. Graus F, Delattre JY, Antoine JC, Dalmau J, Giometto B, Grisold W, et al. Recommended diagnostic criteria for paraneoplastic neurological syndromes. J Neurol Neurosurg Psychiatry 2004;75:1135-40.

9. Irani SR. 'Moonlighting' surface antigens: a paradigm for autoantibody pathogenicity in neurology? Brain 2016;139(Pt 2):304-6.

10. Dalmau J, Rosenfeld MR. Autoimmune encephalitis update. Neuro Oncol 2014;16:771-8.

11. Titulaer MJ, McCracken L, Gabilondo I, Armangué T, Glaser C, lizuka T, et al. Treatment and prognostic factors for long-term outcome in patients with anti-NMDA receptor encephalitis: an observational cohort study. Lancet Neurol 2013;12:157-65.

12. Eker A, Saka E, Dalmau J, Kurne A, Bilen C, Ozen H, et al. Testicular teratoma and anti-N-methyl-D-aspartate receptor-associated encephalitis. J Neurol Neurosurg Psychiatry 2008;79:1082-3.

13. Leypoldt F, Wandinger KP. Paraneoplastic neurological syndromes. Clin Exp Immunol 2014;175:336-48.

14. Yamakura T, Shimoji K. Subunit- and site-specific pharmacology of the NMDA receptor channel. Prog Neurobiol 1999;59:279-98.

15. MacDonald JF, Jackson MF, Beazely MA. Hippocampal long-term synaptic plasticity and signal amplification of NMDA receptors. Crit Rev Neurobiol 2006;18:71-84.

16. Cull-Candy S, Brickley S, Farrant M. NMDA receptor subunits: diversity, development and disease. Curr Opin Neurobiol 2001;11:327-35.

17. Hughes EG, Peng X, Gleichman AJ, Lai M, Zhou L, Tsou R, et al. Cellular and synaptic mechanisms of anti-NMDA receptor encephalitis. J Neurosci 2010;30:5866-75.

18. Armangue T, Titulaer MJ, Málaga I, Bataller L, Gabilondo I, Graus F, et al. Pediatric anti-N-methyl-D-aspartate receptor encephalitis-clinical analysis and novel findings in a series of 20 patients. J Pediatr 2013;162:850-6.e2.

19. Goldberg EM, Titulaer M, de Blank PM, Sievert A, Ryan N. Anti-Nmethyl-D-aspartate receptor-mediated encephalitis in infants and toddlers: case report and review of the literature. Pediatr Neurol 2014;50: 181-4.

20. Zhang L, Wu MQ, Hao ZL, Chiang SM, Shuang K, Lin MT, et al. Clinical characteristics, treatments, and outcomes of patients with anti-N-methyld-aspartate receptor encephalitis: A systematic review of reported cases. Epilepsy Behav 2017;68:57-65.

21. Viaccoz A, Desestret V, Ducray F, Picard G, Cavillon G, Rogemond V, et al. Clinical specificities of adult male patients with NMDA receptor antibodies encephalitis. Neurology 2014;82:556-63.

22. Zhang Y, Liu G, Jiang MD, Li LP, Su YY. Analysis of electroencephalogram characteristics of anti-NMDA receptor encephalitis patients in China. Clin Neurophysiol 2017;128:1227-33.3.

23. Schmitt SE, Pargeon K, Frechette ES, Hirsch LJ, Dalmau J, Friedman D. Extreme delta brush: a unique EEG pattern in adults with anti-NMDA receptor encephalitis. Neurology 2012;79:1094-100.

24. Bacchi S, Franke K, Wewegama D, Needham E, Patel S, Menon D. et al. Magnetic resonance imaging and positron emission tomography in antiNMDA receptor encephalitis: A systematic review. J Clin Neurosci 2018; 52:54-9.

25. Prüss H, Dalmau J, Harms L, Höltje M, Ahnert-Hilger G, Borowski K, et al. Retrospective analysis of NMDA receptor antibodies in encephalitis of unknown origin. Neurology 2010;75:1735-9.

26. Suppiej A, Nosadini M, Zuliani L, Pelizza MF, Toldo I, Bertossi C, et al. Plasma exchange in pediatric anti-NMDAR encephalitis: a systematic review. Brain Dev 2016;38:613-22.

27. Fukata Y, Lovero KL, Iwanaga T, Watanabe A, Yokoi N, Tabuchi K, et al. Disruption of LGI1-linked synaptic complex causes abnormal synaptic transmission and epilepsy. Proc Natl Acad Sci US A 2010;107:3799-804.

28. Ohkawa T, Fukata Y, Yamasaki M, Miyazaki T, Yokoi N, Takashima H, et al. Autoantibodies to epilepsy-related LGI1 in limbic encephalitis neutralize LGI1-ADAM22 interaction and reduce synaptic AMPA receptors. JNeurosci 2013;33:18161-74.

39. Petit-Pedrol M, Sell J, Planagumà J, Mannara F, Radosevic M, Haselmann $\mathrm{H}$, et al. LGI1 antibodies alter Kv1.1 and AMPA receptors changing synaptic excitability, plasticity and memory. Brain 2018;141:3144-59.

30. Ariño H, Armangué T, Petit-Pedrol M, Sabater L, Martinez-Hernandez E, Hara M, et al. Anti-LGI1-associated cognitive impairment: Presentation and long-term outcome. Neurology 2016;87:759-65.

31. Irani SR, Michell AW, Lang B, Pettingill P, Waters P, Johnson MR, et al. Faciobrachial dystonic seizures precede Lgi1 antibody limbic encephalitis. Ann Neurol 2011;69:892-900.

32. Thompson J, Bi M, Murchison AG, Makuch M, Bien CG, Chu K, et al. The importance of early immunotherapy in patients with faciobrachial dystonic seizures. Brain 2018;141:348-56.

33. Navarro V, Kas A, Apartis E, Chami L, Rogemond V, Levy P, et al. Motor cortex and hippocampus are the two main cortical targets in LGI1antibody encephalitis. Brain 2016;139(Pt 4):1079-93.

34. Irani SR, Alexander S, Waters P, Kleopa KA, Pettingill P, Zuliani L, et al. 
Antibodies to Kv1 potassium channel-complex proteins leucine-rich, glioma inactivated 1 protein and contactin-associated protein- 2 in limbic encephalitis, Morvan's syndrome and acquired neuromyotonia. Brain 2010;133:2734-48.

35. Irani SR, Gelfand JM, Bettcher BM, Singhal NS, Geschwind MD. Effect of rituximab in patients with leucine-rich, glioma-inactivated 1 antibodyassociated encephalopathy. JAMA Neurol 2014;71:896-900.

36. Finke C, Prüss H, Heine J, Reuter S, Kopp UA, Wegner F, et al. Evaluation of cognitive deficits and structural hippocampal damage in encephalitis with leucine-rich, glioma-inactivated 1 antibodies. JAMA Neurol 2017; 74:50-9.

37. Lancaster E, Huijbers MG, Bar V, Boronat A, Wong A, MartinezHernandez E, et al. Investigations of caspr2, an autoantigen of encephalitis and neuromyotonia. Ann Neurol 2011;69:303-11.

38. Joubert B, Saint-Martin M, Noraz N, Picard G, Rogemond V, Ducray F, et al. Characterization of a subtype of autoimmune encephalitis with anticontactin-associated protein-like 2 antibodies in the cerebrospinal fluid, prominent limbic symptoms, and seizures. JAMA Neurol 2016;73:111524.

39. van Sonderen A, Ariño H, Petit-Pedrol M, Leypoldt F, Körtvélyessy P, Wandinger KP, et al. The clinical spectrum of Caspr2 antibody-associated disease. Neurology 2016;87:521-8.

40. Maat P, de Beukelaar JW, Jansen C, Schuur M, van Duijn CM, van Coevorden $\mathrm{MH}$, et al. Pathologically confirmed autoimmune encephalitis in suspected Creutzfeldt-Jakob disease. Neurol Neuroimmunol Neuroinflamm 2015;2:e178.

41. Klein CJ, Lennon VA, Aston PA, McKeon A, O'Toole O, Quek A, et al. Insights from LGI1 and CASPR2 potassium channel complex autoantibody subtyping. JAMA Neurol 2013;70:229-34.

42. Lilleker JB, Jones MS, Mohanraj R. VGKC complex antibodies in epilepsy: diagnostic yield and therapeutic implications. Seizure 2013;22: 776-9.

43. Pon NC, Houck KM, Muscal E, Idicula SA. Voltage-gated potassium channel antibody autoimmune encephalopathy presenting with isolated psychosis in an adolescent. J Psychiatr Pract 2017;23:441-5.

44. Benarroch EE. GABAB receptors: structure, functions, and clinical implications. Neurology 2012;78:578-84.

45. Petit-Pedrol M, Armangue T, Peng X, Bataller L, Cellucci T, Davis R, et al. Encephalitis with refractory seizures, status epilepticus, and antibodies to the GABAA receptor: a case series, characterisation of the antigen, and analysis of the effects of antibodies. Lancet Neurol 2014;13:276-86.

46. Spatola M, Petit-Pedrol M, Simabukuro MM, Armangue T, Castro FJ, Barcelo Artigues MI, et al. Investigations in GABAA receptor antibodyassociated encephalitis. Neurology 2017;88:1012-20.

47. Nikolaus M, Knierim E, Meisel C, Kreye J, Prüss H, Schnabel D, et al. Severe GABAA receptor encephalitis without seizures: a paediatric case successfully treated with early immunomodulation. Eur J Paediatr Neurol 2018;22:558-62.

48. Höftberger R, Titulaer MJ, Sabater L, Dome B, Rózsás A, Hegedus B, et al. Encephalitis and GABAB receptor antibodies: novel findings in a new case series of 20 patients. Neurology 2013;81:1500-6.

49. Kruer MC, Hoeftberger R, Lim KY, Coryell JC, Svoboda MD, Woltjer $\mathrm{RL}$, et al. Aggressive course in encephalitis with opsoclonus, ataxia, chorea, and seizures: the first pediatric case of $\gamma$-aminobutyric acid type B receptor autoimmunity. JAMA Neurol 2014;71:620-3.

50. Lai M, Hughes EG, Peng X, Zhou L, Gleichman AJ, Shu H, et al. AMPA receptor antibodies in limbic encephalitis alter synaptic receptor location.
Ann Neurol 2009;65:424-34.

51. Höftberger R, van Sonderen A, Leypoldt F, Houghton D, Geschwind M, Gelfand J, et al. Encephalitis and AMPA receptor antibodies: novel findings in a case series of 22 patients. Neurology 2015;84:2403-12.

52. Zeilhofer HU, Wildner H, Yévenes GE. Fast synaptic inhibition in spinal sensory processing and pain control. Physiol Rev 2012;92:193-235.

53. Thomas RH, Chung SK, Wood SE, Cushion TD, Drew CJ, Hammond $\mathrm{CL}$, et al. Genotype-phenotype correlations in hyperekplexia: apnoeas, learning difficulties and speech delay. Brain 2013;136(Pt 10):3085-95.

54. Iizuka T, Leite MI, Lang B, Waters P, Urano Y, Miyakawa S, et al. Glycine receptor antibodies are detected in progressive encephalomyelitis with rigidity and myoclonus (PERM) but not in saccadic oscillations. J Neurol 2012;259:1566-73.

55. Carvajal-González A, Leite MI, Waters P, Woodhall M, Coutinho E, Balint $\mathrm{B}$, et al. Glycine receptor antibodies in PERM and related syndromes: characteristics, clinical features and outcomes. Brain 2014;137(Pt 8): 2178-92.

56. Kanaani J, Cianciaruso C, Phelps EA, Pasquier M, Brioudes E, Billestrup $\mathrm{N}$, et al. Compartmentalization of GABA synthesis by GAD67 differs between pancreatic beta cells and neurons. PLoS One 2015;10:e0117130.

57. Tüzün E, Dalmau J. Limbic encephalitis and variants: classification, diagnosis and treatment. Neurologist 2007;13:261-71.

58. Malter MP, Helmstaedter C, Urbach H, Vincent A, Bien CG. Antibodies to glutamic acid decarboxylase define a form of limbic encephalitis. Ann Neurol 2010;67:470-8.

59. Ben Achour N, Ben Younes T, Rebai I, Ben Ahmed M, Kraoua I, Ben Youssef-Turki I. Severe dysautonomia as a main feature of anti-GAD encephalitis: report of a paediatric case and literature review. Eur J Paediatr Neurol 2018;22:548-51.

60. Vincent A, Bien CG, Irani SR, Waters P. Autoantibodies associated with diseases of the CNS: new developments and future challenges. Lancet Neurol 2011;10:759-72.

61. Lawn ND, Westmoreland BF, Kiely MJ, Lennon VA, Vernino S. Clinical, magnetic resonance imaging, and electroencephalographic findings in paraneoplastic limbic encephalitis. Mayo Clin Proc 2003;78:1363-8.

62. Perucca P, Dubeau F, Gotman J. Intracranial electroencephalographic seizure-onset patterns: effect of underlying pathology. Brain 2014;137(Pt 1):183-96.

63. Hacohen Y, Wright S, Waters P, Agrawal S, Carr L, Cross H, et al. Paediatric autoimmune encephalopathies: clinical features, laboratory investigations and outcomes in patients with or without antibodies to known central nervous system autoantigens. J Neurol Neurosurg Psychiatry 2013;84: 748-55.

64. Escudero D, Guasp M, Ariño H, Gaig C, Martínez-Hernández E, Dalmau J, et al. Antibody-associated CNS syndromes without signs of inflammation in the elderly. Neurology 2017;89:1471-5.

65. Darnell RB, Posner JB. A new cause of limbic encephalopathy. Brain 2005;128(Pt 8):1745-6.

66. Bascić-Kes V, Kes P, Zavoreo I, Lisak M, Zadro L, Corić L, et al. Guidelines for the use of intravenous immunoglobulin in the treatment of neurologic diseases. Acta Clin Croat 2012;51:673-83.

67. Dogan Onugoren M, Golombeck KS, Bien C, Abu-Tair M, Brand M, Bulla-Hellwig $\mathrm{M}$, et al. Immunoadsorption therapy in autoimmune encephalitides. Neurol Neuroimmunol Neuroinflamm 2016;3:e207.

68. Platt MP, Agalliu D, Cutforth T. Hello from the other side: how autoantibodies circumvent the blood-brain barrier in autoimmune encephalitis. Front Immunol 2017;8:442. 